\title{
Existence of Positive Solutions of Lotka-Volterra Competition Model with Nonlinear Boundary Conditions
}

\author{
Yang Zhang, Mingxin Wang, and Yuwen Wang \\ Natural Science Research Center, Harbin Institute of Technology, Harbin 150080, China \\ Correspondence should be addressed to Yang Zhang; zhangyanghit830217@163.com
}

Received 8 March 2014; Accepted 18 May 2014; Published 26 May 2014

Academic Editor: Dragos-Patru Covei

Copyright ( 2014 Yang Zhang et al. This is an open access article distributed under the Creative Commons Attribution License, which permits unrestricted use, distribution, and reproduction in any medium, provided the original work is properly cited.

\begin{abstract}
A Lotka-Volterra competition model with nonlinear boundary conditions is considered. First, by using upper and lower solutions method for nonlinear boundary problems, we investigate the existence of positive solutions in weak competition case. Next, we prove that $-d_{1} \Delta u=u\left(a_{1}-b_{1} u-c_{1} v\right), x \in \Omega ;-d_{2} \Delta v=v\left(a_{2}-b_{2} u-c_{2} v\right), x \in \Omega ; \partial u / \partial v+f(u)=0, x \in \partial \Omega ; \partial v / \partial v+g(v)=0, x \in \partial \Omega$, has no positive solution when one of the diffusion coefficients is sufficiently large.
\end{abstract}

\section{Introduction}

In this paper, we study the existence of positive solutions to the following problem with nonlinear boundary conditions:

$$
\begin{gathered}
-d_{1} \Delta u=u\left(a_{1}-b_{1} u-c_{1} v\right), \quad x \in \Omega, \\
-d_{2} \Delta v=v\left(a_{2}-b_{2} u-c_{2} v\right), \quad x \in \Omega, \\
\frac{\partial u}{\partial v}+f(u)=0, \quad x \in \partial \Omega, \\
\frac{\partial v}{\partial v}+g(v)=0, \quad x \in \partial \Omega .
\end{gathered}
$$

Here,

(H1) $\Omega \subset \mathbb{R}^{N}(N \geq 1)$ is an open bounded domain, and $v$ is the outward unit normal vector of the boundary $\partial \Omega$;

(H2) for $i=1,2, a_{i}, b_{i}, c_{i}$, and $d_{i}$ are positive constants;

(H3) $f$ and $g$ are strictly increasing $C^{2}$ functions in $\mathbb{R}$ and $f(0)=g(0)=f^{\prime}(0)=g^{\prime}(0)=0$

(H4) $b_{1} / b_{2}>a_{1} / a_{2}>c_{1} / c_{2}$.

In the usual interpretation of the competition model, $u(x)$ and $v(x)$ are population variables; it is natural to consider only nonnegative solutions of (1). There is clearly a trivial solution $u=v=0$ for all values of the parameters. In addition, for some values of parameters, there exist two semitrivial solutions $(u, v)=(u, 0)$ and $(0, v)$. More interesting are the socalled positive solutions or coexistence solutions, where both $u(x)$ and $v(x)$ are positive for all $x \in \Omega$.

By using the positive operator theory, Ahn and Li [1] proved the existence of positive solutions to the following elliptic system:

$$
\begin{gathered}
-d_{1} \Delta u=u f(u, v), \quad x \in \Omega, \\
-d_{2} \Delta v=v g(u, v), \quad x \in \Omega, \\
\frac{\partial u}{\partial v}+\alpha(u)=0, \quad x \in \partial \Omega, \\
\frac{\partial v}{\partial v}+\beta(v)=0, \quad x \in \partial \Omega,
\end{gathered}
$$

where $f(u, v), g(u, v)$ are $C^{1}$ functions in $u$ and $v, f_{u} \leq 0$, $f_{v} \geq 0, g_{u} \leq 0$, and $g_{v} \geq 0$ for $(u, v) \in \mathbb{R}^{+} \times \mathbb{R}^{+} ; \alpha$ and $\beta$ are increasing functions in $\mathbb{R}$ and $\alpha(0)=\beta(0)=0$.

The main project of our paper is to investigate the existence of positive solutions to the problem (1). In Section 2 we state some known results, which are useful throughout this paper. Section 3 is devoted to proving the existence of positive solutions by using the upper and lower solutions method. When the diffusion coefficient $d_{1}$ or $d_{2}$ is sufficiently large, we will prove that problem (1) has no positive solution. 
For the homogeneous Neumann boundary conditions, that is, $f(u) \equiv 0$ and $g(v) \equiv 0$, problem (1) has been studied intensively by many authors. For the related results, please refer to, for instance, [2-8], [9, Section 4.3], and the references cited therein.

\section{Preliminaries}

In this section, we will introduce some notations and lemmas, which serve as the basic tools for the arguments to prove our results.

Throughout this paper, we will consider the solutions $u, v \in C(\bar{\Omega})$. For a given continuous function $q: \bar{\Omega} \rightarrow$ $R$, let $\sigma_{1}(q, d)$ be the principal eigenvalue of the following eigenvalue problem:

$$
\begin{gathered}
-d \Delta u+q(x) u=\lambda u, \quad x \in \Omega, \\
\frac{\partial u}{\partial \nu}=0, \quad x \in \partial \Omega .
\end{gathered}
$$

When the diffusion coefficient $d=1$, we denote the first eigenvalue for (3) by $\sigma_{1}(q)$.

The variational characterization of $\sigma_{1}(d, q)$ is

$$
\begin{aligned}
& \sigma_{1}(d, q) \\
& =\inf \left\{\int_{\Omega}\left(d|\nabla \psi|^{2}+q(x) \psi^{2}\right) d x\right. \\
& \left.: \int_{\Omega} \psi^{2} d x=1, \psi \in H_{1}(\Omega)\right\} .
\end{aligned}
$$

We are concerned with the relation between the sign of $\sigma_{1}(d, q)$ and the function $q$.

Lemma 1 (see [9]). The first eigenvalue $\sigma_{1}(d, q)$ of $(3)$ has the following properties:

$$
\int_{\Omega} q(x) d x \leq 0 \Longrightarrow \sigma_{1}(d, q)<0, \quad \forall d>0 .
$$

Lemma 2 (see [10]). Suppose that $q(x) \in C(\bar{\Omega})$ and $M$ is a positive constant satisfying $M-q(x)>0$. Then the following hold:

(i) $\sigma_{1}(q)<0 \Rightarrow r\left[(M-\Delta)^{-1}(M-q(x))\right]>1$;

(ii) $\sigma_{1}(q)>0 \Rightarrow r\left[(M-\Delta)^{-1}(M-q(x))\right]<1$;

(iii) $\sigma_{1}(q)=0 \Rightarrow r\left[(M-\Delta)^{-1}(M-q(x))\right]=1$.

Next, we consider the nonlinear elliptic problem:

$$
\begin{aligned}
& -\Delta u=E(x, u), \quad x \in \Omega, \\
& \frac{\partial u}{\partial v}+f(u)=0, \quad x \in \partial \Omega,
\end{aligned}
$$

where $f \in C^{2}(\mathbb{R})$ is strictly increasing with $f(0)=0$.

Definition 3. Let $E(x, \xi) \in C(\bar{\Omega} \times \mathbb{R})$ be global Lipschitz continuous in $\xi$ for all $x \in \bar{\Omega}$. The functions $\bar{u}, \underline{u} \in C^{2}(\Omega) \cap C^{1}(\bar{\Omega})$ are called the upper and lower solutions of (6), if $\bar{u}$ and $\underline{u}$ satisfy

$$
\begin{array}{ll}
-\Delta \bar{u} \geq E(x, \bar{u}), & x \in \Omega, \\
\frac{\partial \bar{u}}{\partial \nu}+f(\bar{u}) \geq 0, & x \in \partial \Omega, \\
-\Delta \underline{u} \leq E(x, \underline{u}), & x \in \Omega, \\
\frac{\partial \underline{u}}{\partial \nu}+f(\underline{u}) \leq 0, \quad x \in \partial \Omega .
\end{array}
$$

By using the upper and lower solutions method, the following result was obtained by Ahn and Li [1].

Lemma 4. Suppose that $\bar{u} \geq \underline{u} \geq 0$ are upper and lower solutions of (6); then there exists a maximal solution $\tilde{u}$ of (6) such that $\bar{u} \geq \tilde{u} \geq \underline{u}$.

Lemma 5 (see [1]). Let $P, d$ be positive constants and $h \in$ $C(\bar{\Omega})$. Consider

$$
\begin{aligned}
& -d \Delta u+P u=h, \quad x \in \Omega, \\
& \frac{\partial u}{\partial v}+f(u)=0, \quad x \in \partial \Omega,
\end{aligned}
$$

where $f \in C^{2}(\mathbb{R}), f(0)=0$, and $f$ is strictly increasing. Then the following hold:

(i) problem (8) has a solution $u \in W^{2, m}(\Omega) \cap C^{1, \alpha}(\bar{\Omega})$ for some $\alpha \in(0,1)$, and

$$
\|u\|_{W^{2, m}} \leq C_{0}\|h\|_{\infty}
$$

where $C_{0}$ is dependent on $P$;

(ii) if $0 \neq \equiv \geq 0$, then (8) has a unique positive solution.

Now we consider the following nonlinear boundary value problem:

$$
\begin{aligned}
& -\Delta u=u F(x, u), \quad x \in \Omega, \\
& \frac{\partial u}{\partial \nu}+f(u)=0, \quad x \in \partial \Omega .
\end{aligned}
$$

Lemma 6. Let $f \in C^{2}(\mathbb{R})$ be an increasing and convex function in $\mathbb{R}^{+}$, and satisfy with $f(0)=f^{\prime}(0)=0$. Assume that the function $F$ satisfies the following:

(i) $F \in C\left(\bar{\Omega} \times \mathbb{R}^{+}\right), F(x, u)$ is Lipschitz-continuous in $u$, and the Lipschitz constant $C$ is independent of $(x, u) \in$ $\Omega \times \mathbb{R}^{+}$

(ii) $F(x, u)$ is decreasing in $u$;

(iii) $F(x, 0)>0$ for $x \in \Omega, F(x, u)<0$ in $\Omega \times\left(c_{0}, \infty\right)$ for some constant $c_{0}>0$.

If $\sigma_{1}(-F(x, 0))<0$, then (10) has a unique positive solution. If $\sigma_{1}(-F(x, 0)) \geq 0$, then $u=0$ is the only nonnegative solution of (10). 
Proof. Let $\psi>0$ be the eigenfunction corresponding to the eigenvalue $\sigma_{1}(0)=0$ of problem (3); we can obtain $F(x, \xi)<\sigma_{1}$ when $\xi>c_{0}$, due to the hypothesis (iii). With the assumptions on the function $f$ in mind, for a large $M>0$, we have

$$
\begin{gathered}
-\Delta M \psi-M \psi F(x, M \psi) \geq 0, \quad x \in \Omega, \\
\frac{\partial M \psi}{\partial \nu}+f(M \psi) \geq 0, \quad x \in \partial \Omega .
\end{gathered}
$$

Therefore $T(M \psi) \leq M \psi$, where the operator $T u:=(-\Delta$. $+P \cdot)(F(x, u) u+P u)$ is defined by the problem (8). $T$ is compact in the positive cone $K \subset C(\bar{\Omega})$ by Lemma 5 . The function $F(x, \cdot) \cdot+P \cdot$ is monotone increasing on $\left[0,\|M \psi\|_{\infty}\right]$ for sufficiently large $P>0$. Therefore $T$ is increasing on the order interval $(0, M \psi)$ with $T(0)=0$. Taking advantage of $[1$, Lemma 2.13], we know $T^{\prime}(0)=(-\Delta+P)[F(x, 0)+P]$. The spectral radius $r\left(T^{\prime}(0)\right)>1$ by Lemma 2 . Then, the result of [11, Theorem 7.6] ensures our conclusion.

Next we show the uniqueness. Suppose $u_{1}$ is a positive solution of (10). Let $\widetilde{u}$ be a maximal solution of (10). We claim that $\widetilde{u}=u_{1}$.

Suppose $\tilde{u} \geq u_{1} \not \equiv \tilde{u}$. Then

$$
\begin{aligned}
0 & <\int_{\partial \Omega}\left[u_{1} f(\tilde{u})-\tilde{u} f\left(u_{1}\right)\right] d S \\
& =\int_{\Omega}\left(-u_{1} \Delta \tilde{u}+\tilde{u} \Delta u_{1}\right) d x \\
& =\int_{\Omega} \tilde{u} u_{1}\left[F(x, \tilde{u})-F\left(x, u_{1}\right)\right] d x \leq 0 .
\end{aligned}
$$

The first integral is positive, as $f$ is convex and $f(0)=0$. The last integral is nonpositive, since $F(x, u)$ is decreasing in $u$. This contradiction demonstrates $\widetilde{u} \equiv u_{1}$. If $\lambda_{1}(-F(x, 0)) \geq 0$, the proof is similar to [1, Lemma 2.17], and we omit it. This completes the proof.

By Lemma 5, we are able to conclude the following result.

Proposition 7. Suppose that $f, g$ are convex functions in $\mathbb{R}^{+}$. Then (1) has two semitrivial solutions $\left(\theta_{1}, 0\right)$ and $\left(0, \theta_{2}\right)$, for some $0<\alpha<1$, where $\theta_{1} \in C^{1, \alpha}(\bar{\Omega})$ is the unique positive solution of

$$
\begin{gathered}
-d_{1} \Delta u=u\left(a_{1}-b_{1} u\right), \quad x \in \Omega, \\
\frac{\partial u}{\partial v}+f(u)=0, \quad x \in \partial \Omega,
\end{gathered}
$$

and $\theta_{2} \in C^{1, \alpha}(\bar{\Omega})$ is the unique positive solution of

$$
\begin{gathered}
-d_{2} \Delta v=v\left(a_{2}-c_{2} v\right), \quad x \in \Omega, \\
\frac{\partial v}{\partial v}+g(v)=0, \quad x \in \partial \Omega .
\end{gathered}
$$

Finally, we cite a strong maximum principle (see [5, Proposition 2.2]).
Lemma 8. Suppose that $\Omega$ is smooth and $g \in C\left(\bar{\Omega} \times \mathbb{R}^{1}\right)$. Assume that $z \in C^{2}(\Omega) \cap C^{1}(\bar{\Omega})$ and satisfies

$$
\begin{gathered}
\Delta z(x)+g(x, z(x)) \geq 0, \quad x \in \Omega, \\
\frac{\partial z}{\partial \nu} \leq 0, \quad x \in \partial \Omega .
\end{gathered}
$$

If $z\left(x_{0}\right)=\max _{\bar{\Omega}} z(x)$, then $g\left(x_{0}, z\left(x_{0}\right)\right) \geq 0$.

\section{Existence and Nonexistence of Positive Solution}

By using the upper-lower solutions argument for nonlinear boundary problems, we first study the existence of positive solutions to (1). Our method is technically and conceptually simple in the proof of existence results involving upperlower solutions hypotheses and Leray-Schauder continuation argument. Next, we prove that problem (1) has no positive solution, if one of the diffusion coefficients is sufficiently large. Finally, we discuss the stability of semitrivial solutions.

We will show that the positive solutions of systems (1) have a priori bound.

Lemma 9. Suppose that $(u, v)$ is a positive solution of (1). Then $u \leq a_{1} / b_{1}$ and $v \leq a_{2} / c_{2}$.

Proof. In view of the first equation of (1), $u$ satisfies

$$
\begin{gathered}
-d_{1} \Delta u-u\left(a_{1}-b_{1} u-c_{1} v\right)=0, \quad x \in \Omega, \\
\frac{\partial u}{\partial v}+f(u)=0, \quad x \in \partial \Omega .
\end{gathered}
$$

As $u$ is continuous on the compact set $\bar{\Omega}$ and $f(u) \geq 0$, thanks to Lemma 8 , it is easy to know $u \leq a_{1} / b_{1}$. In a similar manner, we obtain $v \leq a_{2} / c_{2}$.

Next, we give the definitions of upper and lower solutions to (1).

Definition 10. Assume that $(\bar{u}, \bar{v}),(\underline{u}, \underline{v}) \in C^{1}(\bar{\Omega}) \cap C^{2}(\Omega)$. We called that $(\bar{u}, \bar{v})$ and $(\underline{u}, \underline{v})$ are the coupled upper and lower solutions of $(1)$, if $(\bar{u}, \bar{v})$ and $(\underline{u}, \underline{v})$ satisfy

$$
\begin{gathered}
-d_{1} \Delta \bar{u}-\bar{u}\left(a_{1}-b_{1} \bar{u}-c_{1} \underline{v}\right) \geq 0, \quad x \in \Omega, \\
-d_{2} \Delta \bar{v}-\bar{v}\left(a_{2}-b_{2} \underline{u}-c_{2} \bar{v}\right) \geq 0, \quad x \in \Omega, \\
\frac{\partial \bar{u}}{\partial \nu}+f(\bar{u}) \geq 0, \quad x \in \partial \Omega, \\
\frac{\partial \bar{v}}{\partial \nu}+g(\bar{v}) \geq 0, \quad x \in \partial \Omega, \\
-d_{1} \Delta \underline{u}-\underline{u}\left(a_{1}-b_{1} \underline{u}-c_{1} \bar{v}\right) \leq 0, \quad x \in \Omega, \\
-d_{2} \Delta \underline{v}-\underline{v}\left(a_{2}-b_{2} \bar{u}-c_{2} \underline{v}\right) \leq 0, \quad x \in \Omega, \\
\frac{\partial \underline{u}}{\partial v}+f(\underline{u}) \leq 0, \quad x \in \partial \Omega, \\
\frac{\partial \underline{v}}{\partial v}+g(\underline{v}) \leq 0, \quad x \in \partial \Omega .
\end{gathered}
$$


Theorem 11. Suppose that $(\bar{u}, \bar{v})$ and $(\underline{u}, \underline{v})$ are the coupled upper and lower solutions of $(1)$ and $(\bar{u}, \bar{v}) \geq(\underline{u}, \underline{v})$. Then (1) has at least one solution $(u, v)$ and $(\bar{u}, \bar{v}) \geq(u, v) \geq(\underline{u}, \underline{v})$.

Proof. For any given $W:=\left(w_{1}, w_{2}\right) \in[C(\bar{\Omega})]^{2}$ and a sufficiently large positive constant $M$, let

$$
\begin{aligned}
& F^{1}(x)=w_{1}\left(a_{1}-b_{1} w_{1}-c_{1} w_{2}\right)+M w_{1}, \\
& F^{2}(x)=w_{2}\left(a_{2}-b_{2} w_{1}-c_{2} w_{2}\right)+M w_{2}
\end{aligned}
$$

Consider the following problem:

$$
\begin{gathered}
-d_{1} \Delta u+M u=w_{1}\left(a_{1}-b_{1} w_{1}-c_{1} w_{2}\right)+M w_{1}, \quad x \in \Omega, \\
\frac{\partial u}{\partial v}+f(u)=0, \quad x \in \partial \Omega .
\end{gathered}
$$

Since $F^{1}(x) \in C(\bar{\Omega})$, we see that (19) admits a unique solution $u \in C^{1+\alpha}(\bar{\Omega})$ by Lemma 5 . Similarly, the problem

$$
\begin{gathered}
-d_{2} \Delta v+M v=w_{2}\left(a_{2}-b_{2} w_{1}-c_{2} w_{2}\right)+M w_{2}, \quad x \in \Omega, \\
\frac{\partial v}{\partial v}+g(v)=0, \quad x \in \partial \Omega
\end{gathered}
$$

has a unique solution $v \in C^{1+\alpha}(\bar{\Omega})$. Denote $U=(u, v), \bar{U}=$ $(\bar{u}, \bar{v})$, and $\underline{U}=(\underline{u}, \underline{v})$. We define the ordered interval

$$
A=\left\{U \in[C(\bar{\Omega})]^{2}: \underline{U} \leq U \leq \bar{U}\right\}
$$

and an operator $T: A \rightarrow[C(\bar{\Omega})]^{2}$ by

$$
U=T W \text {. }
$$

Thanks to Lemma 5, we have

$$
\begin{aligned}
& \|u\|_{C^{1, \alpha}(\bar{\Omega})} \leq C\left(\left\|w_{1}\left(a_{1}-b_{1} w_{1}-c_{1} w_{2}\right)\right\|_{\infty}+\left\|w_{1}\right\|_{\infty}\right), \\
& \|v\|_{C^{1, \alpha}(\bar{\Omega})} \leq C\left(\left\|w_{2}\left(a_{2}-b_{2} w_{1}-c_{2} w_{2}\right)\right\|_{\infty}+\left\|w_{2}\right\|_{\infty}\right) .
\end{aligned}
$$

Hence, $T(A)$ is bounded in $[C(\bar{\Omega})]^{2}$.

We claim that $T: A \rightarrow[C(\bar{\Omega})]^{2}$ is a compact operator. To see this, it suffices to prove that the operator $T$ is continuous. Suppose that $W_{n}=\left(w_{1 n}, w_{2 n}\right) \rightarrow W=\left(w_{1}, w_{2}\right)$ in $[C(\bar{\Omega})]^{2}$. Denote

$$
\begin{aligned}
& F_{n}^{1}(x)=w_{1 n}\left(a_{1}-b_{1} w_{1 n}-c_{1} w_{2 n}\right)+M w_{1 n} \\
& F_{n}^{2}(x)=w_{2 n}\left(a_{2}-b_{2} w_{1 n}-c_{2} w_{2 n}\right)+M w_{2 n}
\end{aligned}
$$

Then $F_{n}=\left(F_{n}^{1}, F_{n}^{2}\right) \rightarrow F=\left(F^{1}, F^{2}\right)$ in $[C(\bar{\Omega})]^{2}$. Let $U_{n}=$ $T W_{n}$. By Lemma 5, we obtain

$$
\begin{aligned}
& \left\|u_{n}\right\|_{W^{2, p}(\bar{\Omega})} \leq C\left(\left\|F_{n}^{1}(x)-F^{1}(x)\right\|_{\infty}+\left\|F^{1}(x)\right\|_{\infty}\right), \\
& \left\|v_{n}\right\|_{W^{2, p}(\bar{\Omega})} \leq C\left(\left\|F_{n}^{2}(x)-F^{2}(x)\right\|_{\infty}+\left\|F^{2}(x)\right\|_{\infty}\right) .
\end{aligned}
$$

Therefore, $U_{n} \rightarrow U$ in $\left[W^{2, p}(\Omega)\right]^{2}$. Note that $W^{2, p}(\Omega) \hookrightarrow$ $C^{1+\alpha}(\bar{\Omega})$ is compact; it is deduced that $U_{n} \rightarrow U$ in $\left[C^{1+\alpha}(\bar{\Omega})\right]^{2}$. It is obvious that $U=(u, v)$ is the solution of

$$
\begin{aligned}
&-d_{1} \Delta u+M u=u\left(a_{1}-b_{1} w_{1}-c_{1} w_{2}\right)+M w_{1}, \quad x \in \Omega, \\
&-d_{2} \Delta v+M v=v\left(a_{2}-b_{2} w_{1}-c_{2} w_{2}\right)+M w_{2}, \quad x \in \Omega, \\
& \frac{\partial u}{\partial v}+f(u)=0, \quad x \in \partial \Omega, \\
& \frac{\partial v}{\partial v}+g(v)=0, \quad x \in \partial \Omega .
\end{aligned}
$$

This shows that $T: A \rightarrow[C(\bar{\Omega})]^{2}$ is continuous.

Now, we would like to prove $T(A) \subset A$. Suppose that $W \in$ $A$ and $U=T W$, where $U=(u, v), W=\left(w_{1}, w_{2}\right)$. We first prove $\bar{u} \geq u$. In virtue of $\left(w_{1}, w_{2}\right) \in A$, we have that

$$
\begin{gathered}
-d_{1} \Delta \bar{u}+M \bar{u} \geq \bar{u}\left(a_{1}-b_{1} \bar{u}-c_{1} w_{2}\right)+M \bar{u}, \quad x \in \Omega, \\
\frac{\partial \bar{u}}{\partial \nu}+f(\bar{u}) \geq 0, \quad x \in \partial \Omega .
\end{gathered}
$$

Let $z=\bar{u}-u$. Noting that $\bar{u} \geq w_{1}$ and $u$ satisfies (19), it is obvious that

$$
\begin{aligned}
&-d_{1} \Delta z+M z \geq \bar{u}\left(a_{1}-b_{1} \bar{u}-c_{1} w_{2}\right) \\
&-w_{1}\left(a_{1}-b_{1} w_{1}-c_{1} w_{2}\right) \\
&+M\left(\bar{u}-w_{1}\right) \geq 0, \quad x \in \Omega, \\
& \frac{\partial z}{\partial \nu}+f(\bar{u})-f(u) \geq 0, \quad x \in \partial \Omega .
\end{aligned}
$$

On the contrary, we assume that $\bar{u} \geq u$ is not true. By the strong maximum theory (see [12]), there exists $x_{0} \in \partial \Omega$, such that $\min _{x \in \bar{\Omega}} z(x)=z\left(x_{0}\right)=\bar{u}\left(x_{0}\right)-u\left(x_{0}\right)<0$. Thanks to the Hopf boundary lemma, we know

$$
\left.\frac{\partial z}{\partial \nu}\right|_{x=x_{0}}<0
$$

In view of (28), we get

$$
\left.\frac{\partial z}{\partial \nu}\right|_{x=x_{0}} \geq-\left[f\left(\bar{u}\left(x_{0}\right)\right)-f\left(u\left(x_{0}\right)\right)\right]>0 .
$$

This is a contradiction with (29). Thus $z \geq 0$; that is, $\bar{u} \geq u$. Similarly, $\underline{u} \leq u, \bar{v} \geq v$, and $\underline{v} \leq v$. By the Schauder fixed point theorem, $\bar{T}$ has a fixed point $U$ in $A$. The proof is complete.

Theorem 12. Suppose that $b_{1} / b_{2}>a_{1} / a_{2}>c_{1} / c_{2}$ and $f, g$ are convex functions in $\mathbb{R}^{+}$. Then the problem (1) has at least one positive solution.

Proof. By the assumption $f^{\prime}(0)=g^{\prime}(0)=0$, there exists a constant $\beta>0$ such that

$$
\lim _{s \rightarrow 0} \frac{f(s)}{s^{1+\beta}}=0, \quad \lim _{s \rightarrow 0} \frac{g(s)}{s^{1+\beta}}=0 .
$$


Let $\theta_{1}, \theta_{2} \in C^{1, \alpha}(\bar{\Omega})$ be the unique positive solutions of (13) and (14), respectively. Set $\bar{U}=(\bar{u}, \bar{v})=\left(\theta_{1}, \theta_{2}\right), \underline{U}=$ $(\underline{u}, \underline{v})=\left(\varepsilon^{1+\beta} \theta_{1}+\varepsilon, \varepsilon^{1+\beta} \theta_{2}+\varepsilon\right)$, where $\varepsilon>0$ is sufficiently small. Then $\underline{U} \leq \bar{U}$ as $\theta_{1}$ and $\theta_{2}$ are positive on $\bar{\Omega}$. To prove that $\bar{U}$ and $\underline{U}$ are the coupled upper and lower solutions of (1), it suffices to verify inequalities (17) in Definition 10. Consider the following.

(i) Thanks to $\theta_{1}, \theta_{2}>0$ on $\bar{\Omega}$, the following are obvious provided that $\varepsilon>0$ is sufficiently small:

$$
\begin{array}{cc}
-d_{1} \Delta \bar{u}=\bar{u}\left(a_{1}-b_{1} \bar{u}\right) \geq\left(a_{1}-b_{1} \bar{u}-c_{1} \underline{v}\right), \quad x \in \Omega, \\
-d_{2} \Delta \bar{v}=\left(a_{2}-c_{2} \bar{v}\right) \geq\left(a_{c}-c_{2} \bar{v}-b_{2} \underline{u}\right), \quad x \in \Omega, \\
\frac{\partial \bar{u}}{\partial v}+f(\bar{u})=0, \quad \frac{\partial \bar{v}}{\partial \nu}+g(\bar{v})=0, \quad x \in \partial \Omega .
\end{array}
$$

(ii) By Lemma 8, we have that $\theta_{1}(x) \leq a_{1} / b_{1}$ and $\theta(x) \leq$ $a_{2} / c_{2}$ on $\bar{\Omega}$. In virtue of $a_{1}, a_{2}>0$ and $b_{1} / b_{2}>a_{1} / a_{2}>$ $c_{1} / c_{2}$, a direct computation gives

$$
\begin{aligned}
- & d_{1} \Delta \underline{u}-\left(a_{1}-b_{1} \underline{u}-c_{1} \bar{v}\right) \\
= & -d_{1} \Delta\left(\varepsilon^{1+\beta} \theta_{1}+\varepsilon\right) \\
& -\left(\varepsilon^{1+\beta} \theta_{1}+\varepsilon\right)\left(a_{1}-b_{1}\left(\varepsilon^{1+\beta} \theta_{1}+\varepsilon\right)-c_{1} \theta_{2}\right) \\
= & \varepsilon^{1+\beta}\left[\theta_{1}\left(a_{1}-b_{1} \theta_{1}\right)\right] \\
& -\left(\varepsilon^{1+\beta} \theta_{1}+\varepsilon\right)\left(a_{1}-b_{1}\left(\varepsilon^{1+\beta} \theta_{1}+\varepsilon\right)-c_{1} \theta_{2}\right) \\
= & -\varepsilon\left(a_{1}-c_{1} \theta_{2}\right)+o(\varepsilon) \\
\leq & -\varepsilon\left(\frac{a_{1}-c_{1} a_{2}}{c_{2}}\right)+o(\varepsilon) \\
< & 0, \quad x \in \Omega
\end{aligned}
$$

provided $0<\varepsilon \ll 1$. Since the function $f$ is convex in $\mathbb{R}^{+}$, we know that, when $0<\varepsilon \ll 1$,

$$
\begin{aligned}
\frac{\partial \underline{u}}{\partial \nu}+f(\underline{u})= & \varepsilon^{1+\beta} \frac{\partial \theta_{1}}{\partial \nu}+f\left(\varepsilon^{1+\beta} \theta_{1}+\varepsilon\right) \\
& -\varepsilon^{1+\beta} f\left(\theta_{1}\right)+f\left(\varepsilon^{1+\beta} \theta_{1}+\varepsilon\right) \\
= & \frac{f\left(\varepsilon^{1+\beta} \theta_{1}+\varepsilon\right)}{\left(\varepsilon^{1+\beta} \theta_{1}+\varepsilon\right)^{1+\beta}} \frac{\left(\varepsilon^{1+\beta} \theta_{1}+\varepsilon\right)^{1+\beta}}{\varepsilon^{1+\beta}}-f\left(\theta_{1}\right) \\
\leq & 0, \quad x \in \partial \Omega .
\end{aligned}
$$

Similarly,

$$
\begin{gathered}
-d_{1} \Delta \underline{v}-\left(a_{1}-b_{1} \bar{u}-c_{1} \underline{v}\right)<0, \quad x \in \Omega, \\
\frac{\partial \underline{v}}{\partial \nu}+f(\underline{v}) \leq 0, \quad x \in \partial \Omega .
\end{gathered}
$$

We have proved that $\bar{U}, \underline{U}$ are the coupled upper and lower solutions of (1). Taking advantage of Theorem 11, (1) has at least one positive solution. The proof is complete.

Next, we show that (1) has no positive solution, when the diffusion coefficient $d_{1}$ or $d_{2}$ is sufficiently large.

Theorem 13. There exists a positive constant $M=M\left(a_{i}, b_{i}, c_{i}\right)$ such that when $\max \left\{d_{1}, d_{2}\right\} \geq M$, problem (1) has no positive solution.

Proof. There exists a positive constant $C$, independent of $u$ and $v$, such that

$$
\left\|u-u^{*}\right\|_{\infty} \leq \frac{C}{d_{1}}, \quad\left\|v-v^{*}\right\|_{\infty} \leq \frac{C}{d_{2}},
$$

where

$$
u^{*}=\frac{\int_{\partial \Omega} f(u) u}{\int_{\partial \Omega} f(u)}, \quad v^{*}=\frac{\int_{\partial \Omega} g(v) v}{\int_{\partial \Omega} g(v)} .
$$

Following the results of Lemma 9, we have

$$
\max \left\{\|u\|_{\infty},\|v\|_{\infty}\right\} \leq C \equiv \max \left\{\frac{a_{1}}{b_{1}}, \frac{a_{2}}{b_{2}}\right\} .
$$

Rewrite (1) as

$$
\begin{gathered}
-d_{1} \Delta\left(u-u^{*}\right)=u\left(a_{1}-b_{1} u-c_{1} v\right), \quad x \in \Omega, \\
-d_{2} \Delta\left(v-v^{*}\right)=v\left(a_{2}-b_{2} u-c_{2} v\right), \quad x \in \Omega, \\
\frac{\partial\left(u-u^{*}\right)}{\partial v}+f(u)=0, \quad x \in \partial \Omega, \\
\frac{\partial\left(v-v^{*}\right)}{\partial v}+g(v)=0, \quad x \in \partial \Omega .
\end{gathered}
$$

Note that $\left\|u\left(a_{1}-b_{1} u-c_{1} v\right)\right\|_{\infty} \leq C=\max _{0 \leq u, v \leq C} \mid u\left(a_{1}-b_{1} u-\right.$ $\left.c_{1} v\right) \mid$. Multiplying the first equation of (39) by $u-u^{*}$, and integrating on $\Omega$, we derive that, by Green's identity, Holder's inequality, and Poincare's inequality,

$$
\begin{aligned}
\int_{\Omega}|\nabla u|^{2} & \leq \frac{\left|u\left(a_{1}-b_{1} u-c_{1} v\right)\right|}{d_{1}} \int_{\Omega}\left|u-u^{*}\right| \\
& \leq \frac{C}{d_{1}}\left\|u-u^{*}\right\|_{2} \leq \frac{C}{d_{1}}\|\nabla u\|_{2},
\end{aligned}
$$

which implies that

$$
\left\|u-u^{*}\right\|_{2} \leq \frac{C}{d_{1}} .
$$

By Lemma 5, (36), and (41), we obtain

$$
\left\|u-u^{*}\right\|_{W^{2,2}(\Omega)}
$$

$$
\begin{aligned}
& \leq C\left(\left\|u-u^{*}\right\|_{2}+\frac{\left\|u\left(a_{1}-b_{1} u-c_{1} v\right)\right\|_{\infty}}{d_{1}}\right) \\
& \leq \frac{C}{d_{1}} .
\end{aligned}
$$


Thanks to the Sobolev embedding theorem (see [12]), for $0<$ $\beta<1$,

$$
\left\|u-u^{*}\right\|_{C^{1, \beta}(\bar{\Omega})} \leq \frac{C}{d_{1}} .
$$

Review to the third equation of (1), we obtain

$$
|D u||\nu| \geq|D u \cdot v|=f(u) .
$$

In view of (43) and (44), it is easy to see

$$
\frac{C}{d_{1}} \geq f(u)>f\left(u^{*}-\frac{C}{d_{1}}\right) .
$$

Note that $f(u)$ is increasing function and $f(0)=0$, as $d_{1} \rightarrow$ $\infty$, and

$$
0 \geq f\left(u^{*}\right)>0
$$

This is a contradiction. The same proof as above works equally well for the case when $d_{2}$ is large. This completes the proof.

Finally, we discuss the stability of semitrivial solutions.

Theorem 14. Suppose that $f, g$ are convex functions in $\mathbb{R}^{+}$. Then the following hold:

(a) the semitrivial solution $\left(\theta_{1}, 0\right)$ is unstable;

(b) the semitrivial solution $\left(0, \theta_{2}\right)$ is unstable.

Proof. For part (a), to prove the stability of $\left(\theta_{1}, 0\right)$, we consider the corresponding elliptic system eigenvalue problem:

$$
\begin{gathered}
-d_{1} \Delta u-\left(a_{1}-2 b_{1} \theta_{1}\right) u+c_{1} \theta_{1} v=\lambda u, \quad x \in \Omega, \\
-d_{2} \Delta v-\left(a_{2}-b_{2} \theta_{1}\right) v=\lambda v, \quad x \in \Omega, \\
\frac{\partial u}{\partial v}+f^{\prime}\left(\theta_{1}\right) u=0, \quad x \in \partial \Omega, \\
\frac{\partial v}{\partial v}=0, \quad x \in \partial \Omega .
\end{gathered}
$$

First, observe that all eigenvalues of (47) are real since they are also eigenvalues of the second equation:

$$
\begin{gathered}
-d_{2} \Delta v-\left(a_{2}-b_{2} \theta_{1}\right) v=\lambda v, \quad x \in \Omega, \\
\frac{\partial v}{\partial n}=0, \quad x \in \partial \Omega .
\end{gathered}
$$

Next, note that $b_{1} / b_{2}>a_{1} / a_{2}>c_{1} / c_{2}$; by Lemma 1 , we have $\sigma_{1}\left(d_{2}, q\right)<0$. Thus $\left(\theta_{1}, 0\right)$ is unstable. In a similar way, we are able to conclude (b).

\section{Conflict of Interests}

The authors declare that there is no conflict of interests regarding the publication of this paper.

\section{References}

[1] I.-K. Ahn and L.-G. Li, "Positive solutions of certain elliptic systems with density-dependent diffusions," Proceedings of the Royal Society of Edinburgh A, vol. 125, no. 5, pp. 1031-1050, 1995.

[2] M. Iida, T. Muramatsu, H. Ninomiya, and E. Yanagida, "Diffusion-induced extinction of a superior species in a competition system," Japan Journal of Industrial and Applied Mathematics, vol. 15, no. 2, pp. 233-252, 1998.

[3] Y. Kan-on and E. Yanagida, "Existence of nonconstant stable equilibria in competition-diffusion equations," Hiroshima Mathematical Journal, vol. 23, no. 1, pp. 193-221, 1993.

[4] K. Kishimoto and H. F. Weinberger, "The spatial homogeneity of stable equilibria of some reaction-diffusion systems on convex domains," Journal of Differential Equations, vol. 58, no. 1, pp. 1521, 1985.

[5] Y. Lou and W.-M. Ni, "Diffusion, self-diffusion and crossdiffusion," Journal of Differential Equations, vol. 131, no. 1, pp. 79-131, 1996.

[6] H. Matano and M. Mimura, "Pattern formation in competitiondiffusion systems in nonconvex domains," Research Institute for Mathematical Sciences Kyoto University, vol. 19, no. 3, pp. 10491079, 1983.

[7] M. Mimura, S.-I. Ei, and Q. Fang, "Effect of domain-shape on coexistence problems in a competition-diffusion system," Journal of Mathematical Biology, vol. 29, no. 3, pp. 219-237, 1991.

[8] H. Ninomiya, "Separatrices of competition-diffusion equations," Journal of Mathematics of Kyoto University, vol. 35, no. 3, pp. 539-567, 1995.

[9] W.-M. Ni, The Mathematics of Diffusion, vol. 82 of CBMSNSF Regional Conference Series in Applied Mathematics, SIAM, Philadelphia, Pa, USA, 2011.

[10] L. Li, "Coexistence theorems of steady states for predator-prey interacting systems," Transactions of the American Mathematical Society, vol. 305, no. 1, pp. 143-166, 1988.

[11] H. Amann, "Fixed point equations and nonlinear eigenvalue problems in ordered Banach spaces," SIAM Review, vol. 18, no. 4, pp. 620-709, 1976.

[12] D. Gilbarg and N. S. Trudinger, Elliptic Partial Differential Equations of Second Order, Springer, Berlin, Germany, 2nd edition, 1983. 


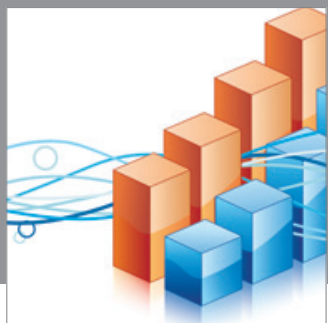

Advances in

Operations Research

mansans

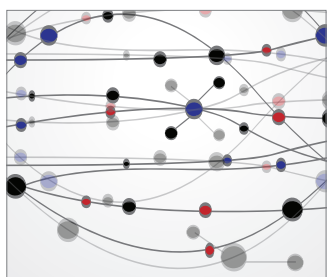

The Scientific World Journal
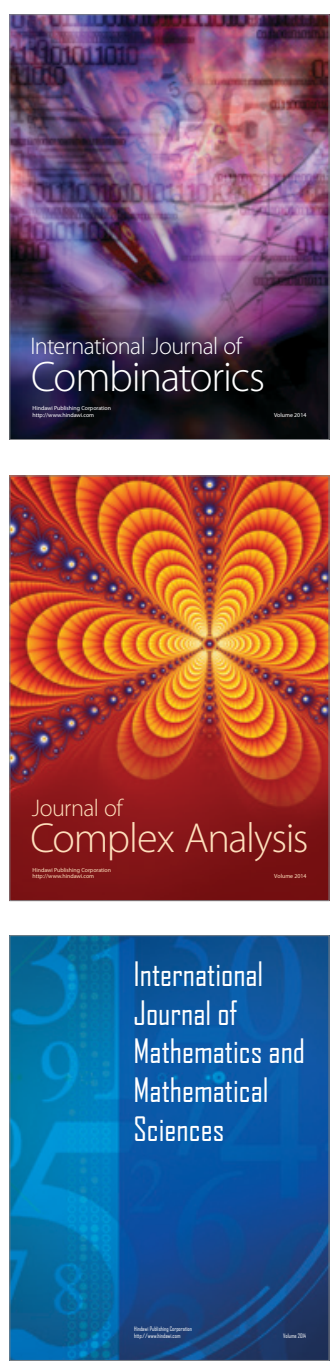
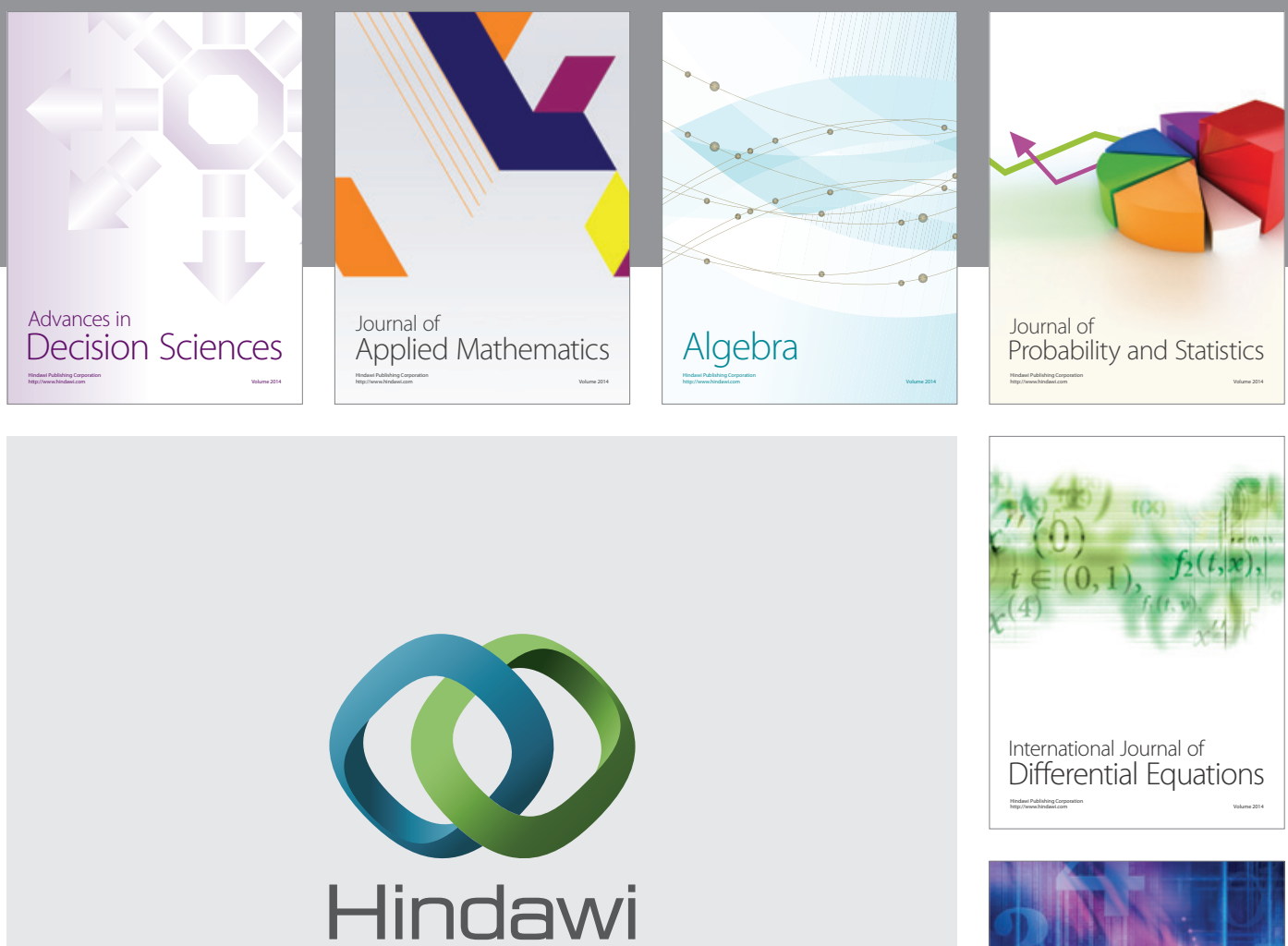

Submit your manuscripts at http://www.hindawi.com
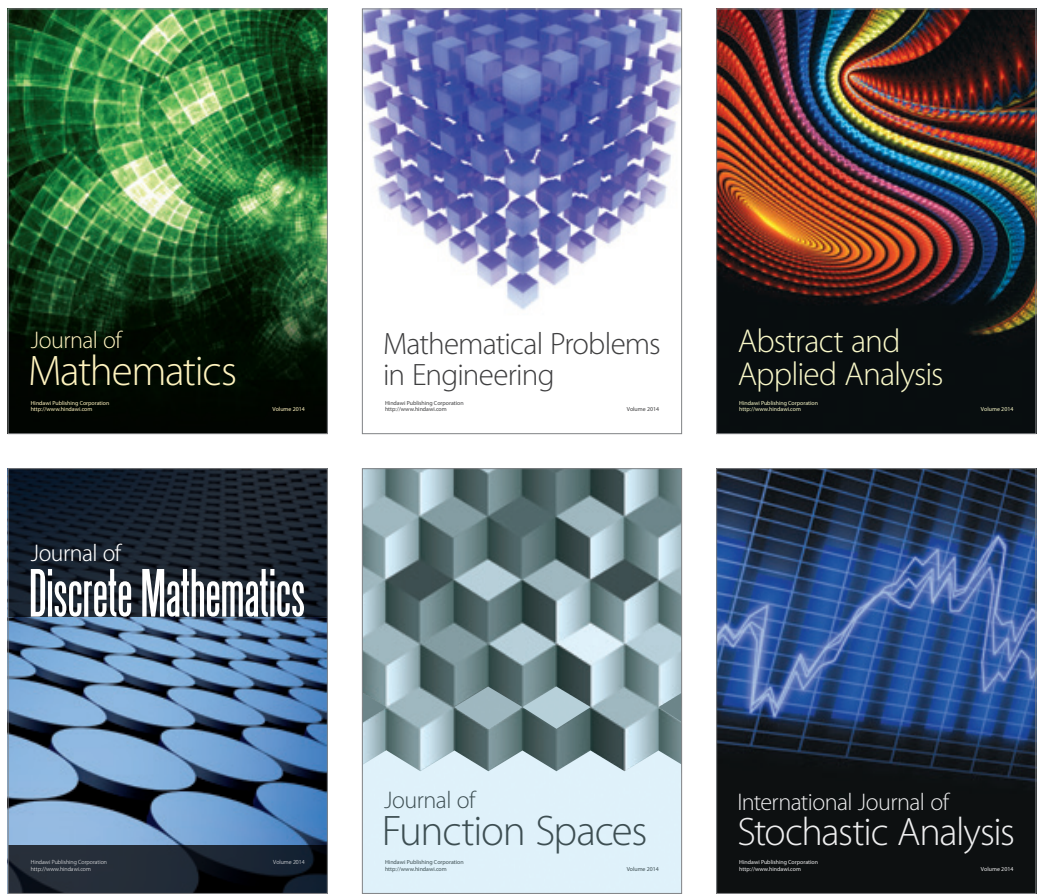

Journal of

Function Spaces

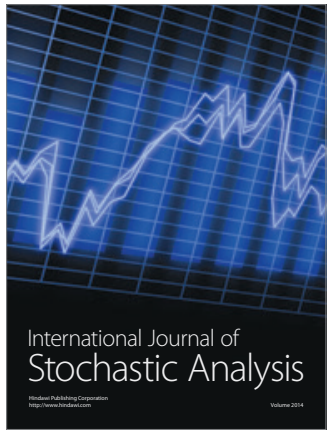

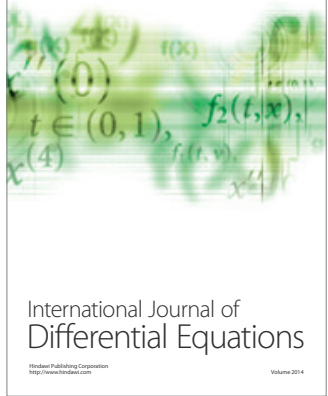
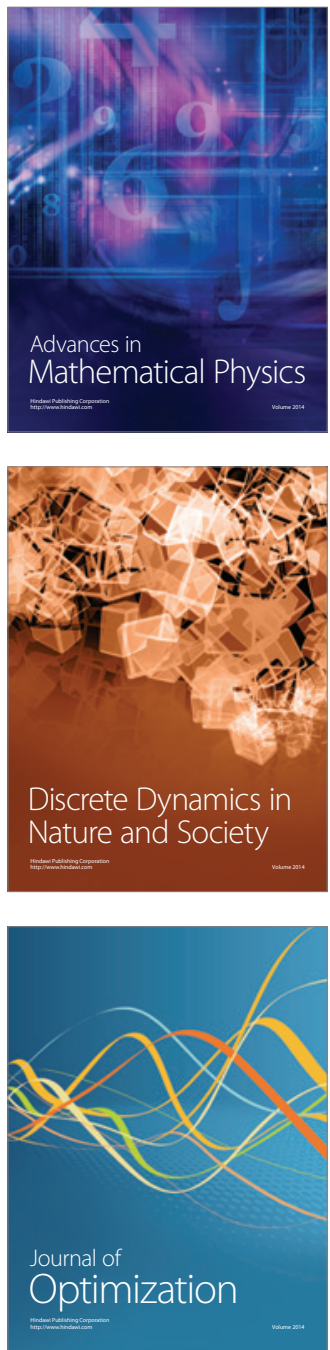